\title{
Health Risk of Obesity in Native American Adolescents
}

\author{
Benjamin D. Knisley ${ }^{1}$, Grace Crosby ${ }^{2}$, Linn Carothers ${ }^{1}$, M. Margaret Barth ${ }^{2}$ \\ ${ }^{1}$ Department of Natural \& Mathematical Sciences, California Baptist University, USA \\ ${ }^{2}$ Department of Health Sciences, Nutrition \& Food Sciences, California Baptist University, USA
}

\begin{tabular}{l}
\hline \hline Article Info \\
\hline Article history: \\
Received Oct 02, 2014 \\
Revised Feb 21, 2015 \\
Accepted May 22, 2015 \\
\hline
\end{tabular}

Keyword:

Adolescents

Anthropometric Indicators

Body Mass Index

Native American

Obesity Prevention

\begin{abstract}
Recent reports demonstrate the need to improve methods for identifying obesity among adolescent minority populations, especially among Native American adolescents. Our study examined several anthropometric measures to see which one was the best indicator of obesity for Native American adolescents. We compared our data with that provided by the Centers for Disease Control and Prevention (CDC), and the national Health and Nutrition Examination Surveys (NHANESIII). We examined which measures differed significantly from the NHANESIII, and which were most effective for measuring obesity in Native American adolescents. Our study population represented a cross-sectional, epidemiological population $(n=183)$ of Native American students (ages 14-18) from diverse tribal backgrounds at an urban residential high school. We obtained baseline anthropometric measurements of height, weight, waist, triceps skin-fold, and calf skin-fold measures from the Native American students and compared this information with reference data to assess weight classification by body mass indices (BMI) for our population. Under the conditions of our study, we found the calf skin-fold measure to be the best indicator of normal weight in male Native American adolescents, and the triceps skin-fold measure to be the best indicator of obesity in male Native American adolescents. The assessment of health risk by anthropometric measures we obtained appears appropriate for predicting obesity and developing effective interventions for Native American adolescents in general. Correlations between anthropometric measures and obesity in our study population may prove of significant interest for monitoring obesity prevention initiatives for Native American adolescents.
\end{abstract}

Copyright (C) 2015 Institute of Advanced Engineering and Science. All rights reserved.

\section{Corresponding Author:}

Benjamin D. Knisley,

Department of Natural \& Mathematical Sciences,

California Baptist University,

8432 Magnolia Avenue, Riverside, CA 92504 USA.

Email: benjamindavid.knisely@calbaptist.edu

\section{INTRODUCTION}

The concept of obesity as a disease, with well-defined complications, is approximately one century old. What has made the problem alarming is the exponential increase in the prevalence and incidence of obesity over the past few decades; this increase has led the World Health Organization (WHO) to declare it a pandemic and worldwide public health crisis [1]. In America alone, 31.8\% of all children and adolescents are now classified as overweight or obese [2].This suggests that the degree of adolescent obesity has increased in recent decades [3]. Furthermore, in three independent studies data reflecting minority populations suggests that underrepresented groups are among those at risk for developing higher rates of overweight and obesity when compared with whites [4],[5]. That is, underrepresented groups and minorities play an inseparable part for us to fully grasp the impact of obesity in our current U.S. population. As shown in Figure 4, we found that male and female cases of obesity made up $38 \%$ of our population ( 70 out of 183 Native American adolescents). The relative frequency of our statistics is approximately double that reported by the CDC (18\%) [6]. 
In addition, a review of the current literature demonstrates that studies on adolescent obesity among minority populations are relatively rare. Information is especially scarce concerning obesity in Native American adolescents. Although some research on obesity has been done among Native American adults from the Pima Indians of Arizona, our study sought to address the unique needs of Native American adolescents using a tribally diverse sample to improve the relevancy of our results to the Native American adolescent population in general.

Currently, most equations that predict obesity are derived from predominantly white sample populations [7]. This does not take into account biological variations between races, and can lead to inaccurate results when these equations are applied to populations from diverse racial backgrounds.

In the world of evidence-based medicine, it is necessary to acknowledge racial differences among our national population in order to provide sufficient evidence for treating minorities. For example, a recent study in 2012 among African American students aged 18-27 described "an unexpected finding revealing that waist circumference was significantly associated with cardiovascular reactivity when analyzed; however, this relationship was no longer significant after BMI was entered into the equation" [8]. Thus, systematic error can result in the inaccurate estimation of the relative body fat of African Americans, and therefore definitions of obesity in minorities, if these inherent differences are ignored.

\section{RESEARCH METHOD}

The objective of our study was to identify prevalent anthropometric indicators of obesity in our Native American adolescent population and to compare these results with national statistics. The primary baseline anthropometric indicators for our research within our Native American population consisted of the waist, triceps skin-fold, and calf skin-fold measures, and were gathered using standardized protocols. Measures such as height, weight, and BMI contained no missing data and utilized all 183 Native American adolescents (ages 14-18). Missing data was recorded within our three primary measures. Our collected data was then compiled and assessed as a pilot study to show which indicators of obesity were creating discrepancies with national data comparisons. All statistical graphs and analyses were generated using R-3.1.0 statistical software [9].

\section{RESULTS AND ANALYSIS}

There were three basic steps in our data analysis. For the first step, we selected two weight categories for comparison, namely, normal (BMI levels between 18.5-24.9) and obese weight categories (BMI greater than or equal to 30.0) to assess which anthropometric indicators were best suited for inference. As shown in Figure 1, we also created a time-series plot of means for both genders to display the variation contained in BMI throughout time. At the age of 14, a large number of male and female adolescents were already classified as overweight. By the age of 18, many of these had moved into the obese weight category. Overall, this figure demonstrates that climbing rates of BMI in both gender categories correspond to rising rates predicted by NHANESIII data [3].

The second step of our data analysis, as shown in Figures 2.0-2.1, was to identify tell-tale signs of normality in our distributions. Using the Shapiro-Wilks test, we assessed waist, triceps skin-fold, and calf skin-fold measures to uncover several degrees of normal bell curve assumptions. From this, we inferred which measure would be best suited to classify normal and obese weight categories. For instance, in figure 2.0 we can observe the approach of normal weight class Native American males approaching bell curve assumptions with the calf mean (i.e., the shape of the distribution in this image is most alike to a standard bell curve). Hence, it appears that calf-mean measures are best suited for predicting normal weight classes for male Native American adolescents. In contrast, the normal weight male triceps-skin fold and waist circumference measures deviate more from normal-bell curve assumptions. In fact, waist circumference measures deviate more than triceps-skin fold measures and suggest waist circumference to be the poorest indicator for predicting normal weight classes for male Native American adolescents. Likewise, with figure 2.1 we observe obese weight classes for male Native Americans and discover once again that waist circumference is the poorest - in terms of its shape - in being matched with a standard bell curve. However, notice the switch between calf and triceps skin-fold measures. For male obese weight categories it appears that the triceps-skin fold measure is the best indicator for predicting obesity among our population due to its relative likeness with a standard bell curve. Later on in this study (see Table 1) we will observe the definitive proof that statistically demonstrates which indicator is most appropriate for accurately predicting obese and normal weight classes for male Native Americans. Until then, these figures give appropriate hints and suggestions that our populations appear to approach normal curve assumptions much more effectively among certain anthropometric indicators. 
Figures 3.0-3.2 show the third step in our data analysis, which was to obtain and analyze side-byside normal weight and obesity density dual-gender plots based on anthropometric measures of waist, triceps skin-fold, and calf skin-fold. The measures were compared with the national measures from NHANESIII. Afterwards, we obtained z-scores to determine which measurements from our Native American adolescents aligned closest with NHANESIII data. Recall the purpose for figures 2.0-2.1 whereby they gave candid hints about the underlying distributions for each anthropometric measure in relation to a standard bell curve. Likewise, Figures 3.0-3.2 provide suggestions about each underlying distribution within each anthropometric measure for male and female Native Americans in relation to normal curve assumptions. However, instead of being primarily concerned with relationships to a relative likeness with standard bell curves we added another feature that visually compares and graphs the mean derived from NHANESIII in each figure. For example, notice the vertical blue and green lines in figure 3.0, these lines represent the weighted mean obtained from our calculations with NHANESIII ${ }^{4}$ for male and female Native Americans respectively. With this information we provided graphs to provide some visual information that suggests how close or far off our weighted means were to means found in each of our anthropometric indicators. For instance, with figure 3.0 notice the proximity of our blue vertical mean (weighted mean equals 83.33 and pooled S.D. of 3.62 for Males) to the mean and S.D. for Native American males when measuring waist circumference (73.30 and S.D. of 17.72). The means by themselves may suggest a disparity between NHANESIII and our Native American populations, however, without a closer look at the underlying distribution we are missing half of the picture. Hence, the density maps shown in figures 3.0-3.2 (e.g., notice that figures 2.0 and 2.1 are included as part of figures 3.0-3.2 in our male categories) illustrate how close or remotely different each distribution is from a standard bell curve. Thus, armed with these dual-gender plots we can easily distinguish which anthropometric measure is much more likely to be akin to a normal NHANESIII distribution. Therefore, we can see that waist circumference once again fails to appear as normally distributed or near (in terms of the mean) to NHANESIII assumptions and paves the way for us to search for other anthropometric indicators that could be much more effective predictors for weight class in our Native American population (see figures 3.1-3.2).

Upon further analyzing the density plots, we found that the waist mean appears to be far from the shape of a normal bell- curve; however, the triceps skin-fold and calf skin-fold means appeared to approach the familiar normal distribution. The waist mean was widely skewed, and served as the poorest indicator for predicting normal or obese weight for male Native American adolescents. This implies that current BMI standards may not be appropriate for assessing normal weight and obesity in Native American adolescents. Furthermore, BMI cutoffs among other minority populations have been shown to create systematic error when assessing gestational diabetes millitus (GDM) [10]. In fact, in one 2014 study headed by M. Hedderson her team stated: "there is ongoing debate surrounding the definition of overweight and obesity in Asian populations: the World Health Organization proposed a BMI cutoff of $23.0 \mathrm{~kg} / \mathrm{m}^{2}$ for overweight among Asians in 2000, compared with a cutoff of $25.0 \mathrm{~kg} / \mathrm{m}^{2}$ for non-Asian populations [10]." That is, the demographic distribution of obesity (highest among African Americans and lowest among Asians) does not mirror the demographic distribution of GDM (lowest among African Americans and highest among Asians) [10]. Thus, racial disparities exist when assessing risk of GDM using standard BMI protocols that do not account for racial differences. Moreover, without the BMI measure clinicians are unable to make sound predictions about who is at risk for GDM without taking racial/ethnic disparities into consideration. In addition, in a 2012 study conducted on 66 African American, 72 Hispanic, and 47 white men and women, aged $\geq 45$ Joan Carroll and her team discovered that Middle-aged and older African-American men and women had lower VAT (visceral adipose tissue) despite similar BMI and waist circumference measurements [11]. That is, altered relationships between anthropometric measures and VAT may have implications for defining metabolic risk in different populations based on race [11]. Therefore, different waist circumference or BMI cutoff points may be necessary to adequately reflect VAT risk in different racial/ethnic groups [11]. Thus, differences in our Native American population appear to approach this same trend and anthropometric measures such as waist circumference fail to meet standards for predicting obese or overweight categories. Consequently, if such measures continue unabated then we will inevitably face skewed results that will create greater schisms when interpreting obese or overweight data among minority populations. Resultantly, the calf skin-fold mean when analyzed for our population appeared to be a much better indicator for predicting normal weight categories for male Native American adolescents. Moreover, the triceps skin-fold mean appeared to be a better indicator for assessment in predicting obese weight categories for male Native American adolescents.

In our male population $35 \%$ of the subjects were classified as obese ( 35 out of 99 males), while in our female population $41 \%$ of the subjects were classified as obese ( 35 out of 84 females). We used our timeseries plot in Figure 3 to identify cases of overweight and obesity trending and climaxing. We found that by the age of 14 both males and females were already classified as overweight with a BMI greater than 25 . 
However, by the age of 18 , significant segments of both populations had moved into the obese category. Waist, triceps skin-fold, and calf skin-fold measures were analyzed for both genders by weight class. Afterwards, we reported the Shapiro-Wilks test results with their respective p-values (listed in Tables 1-3).

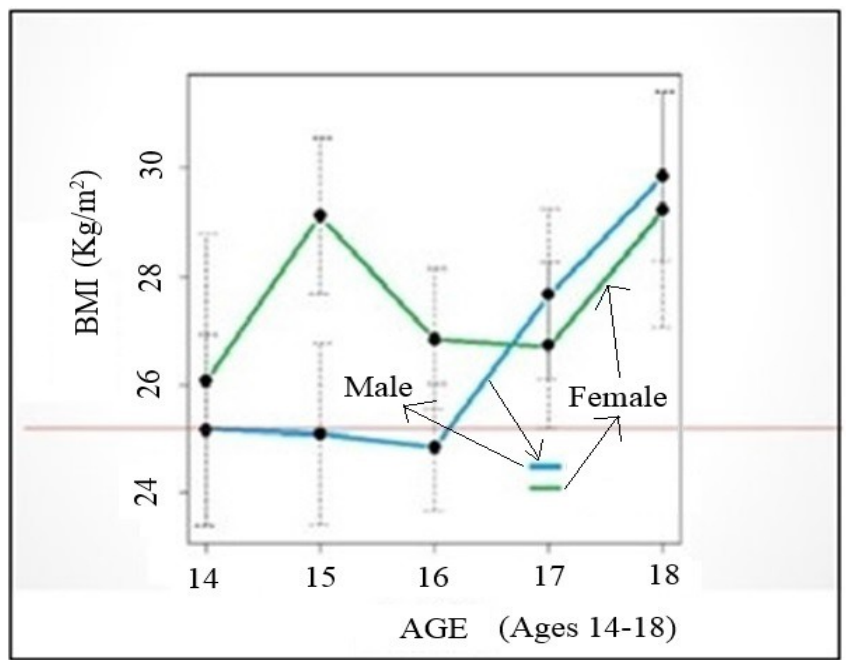

Figure 1. Time-series plot-of-means for male and female Native American adolescents (ages 14-18)

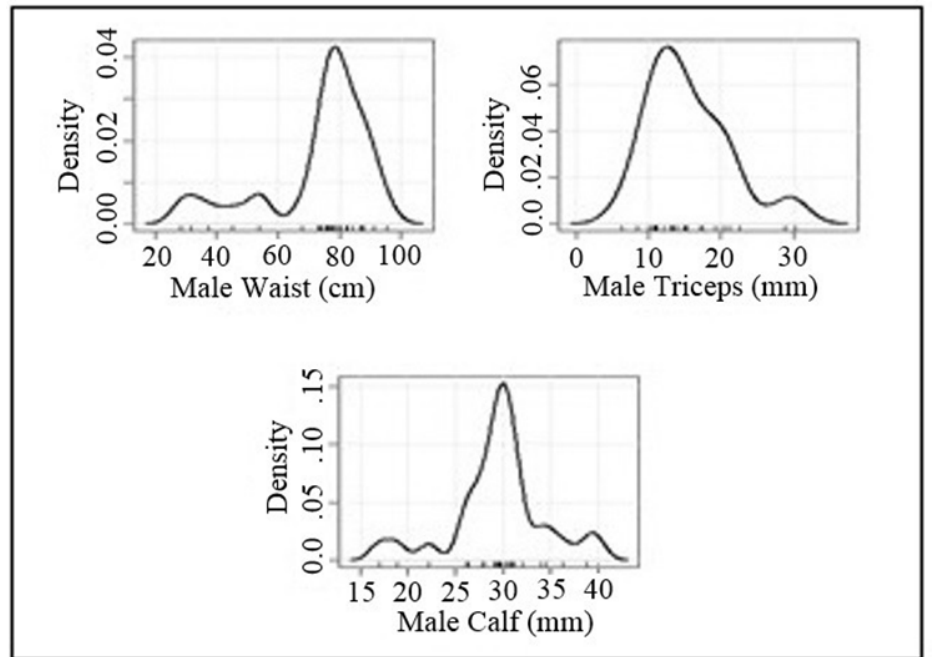

Figure 2. Normal weight class density plots to visualize normality for male Native American adolescents (ages 14-18) 


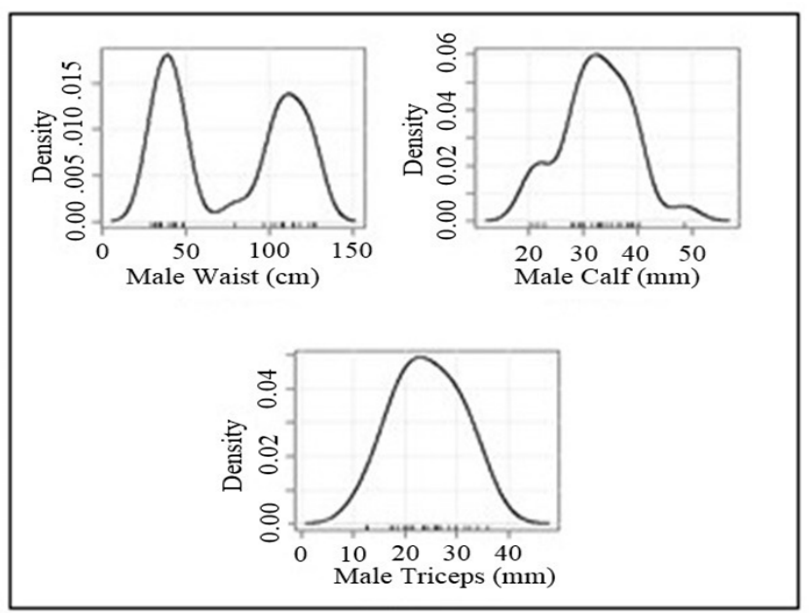

Figure 3. Obese weight class density plots to visualize normality for male Native American adolescents (ages 14-18)

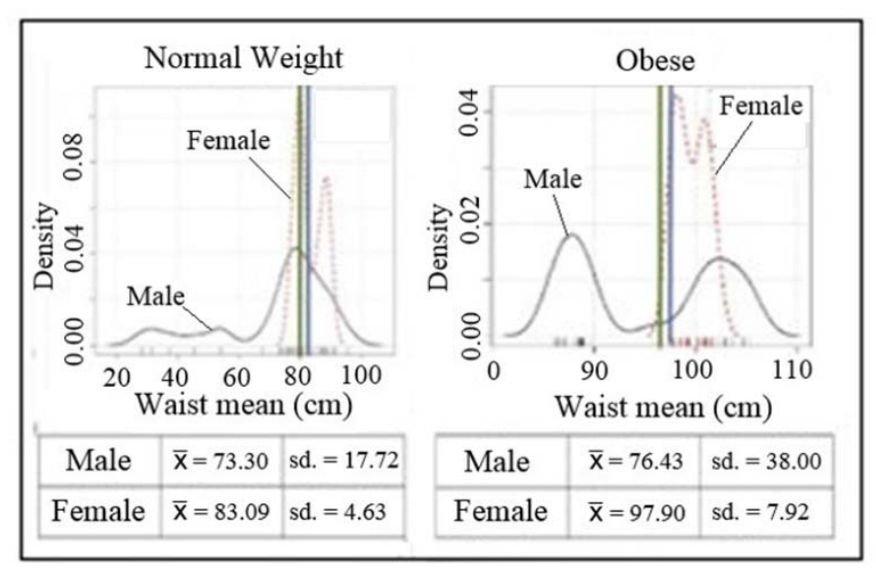

Figure 4. Weight class specific dual-gender density plots visualizing normality and mean proximity to NHANESIII data with waist mean for male and female Native American adolescents (ages 14-18)

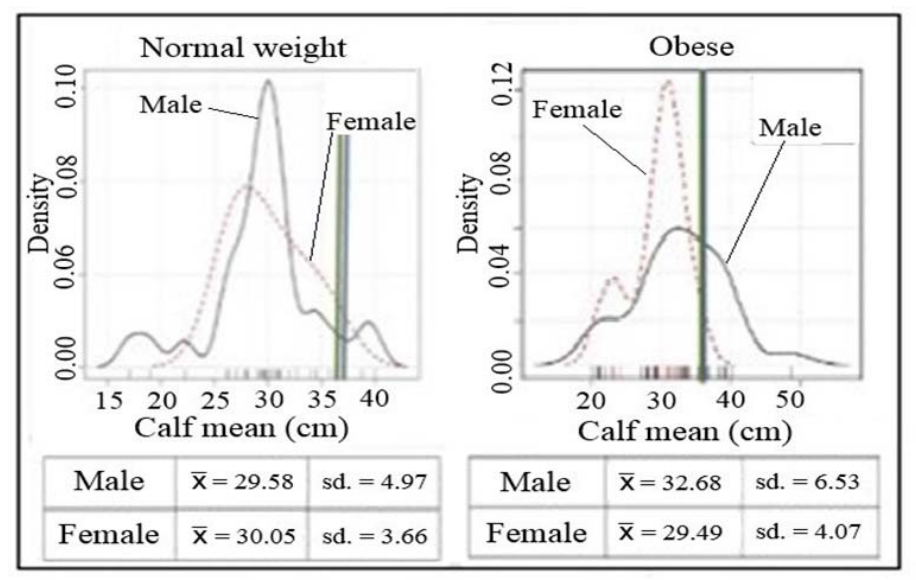

Figure 5. Weight class specific dual-gender density plots visualizing normality and mean proximity to NHANESIII data with calf skinfold mean for both male and female Native American adolescents (ages 14-18) 


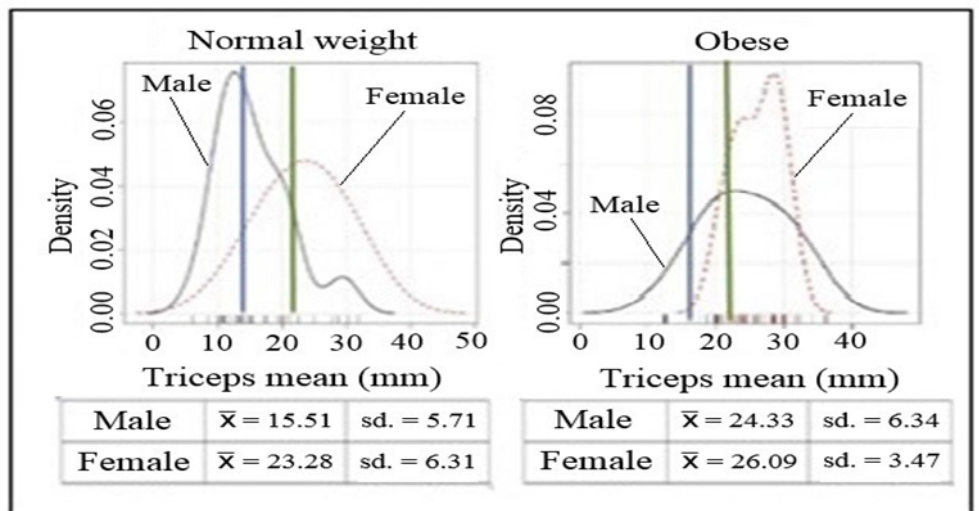

Figure 6. Weight-class specific dual-gender density plots visualizing normality and mean proximity to NHANESIII data with triceps skin-fold mean for male and female Native American adolescents (ages 14-18)

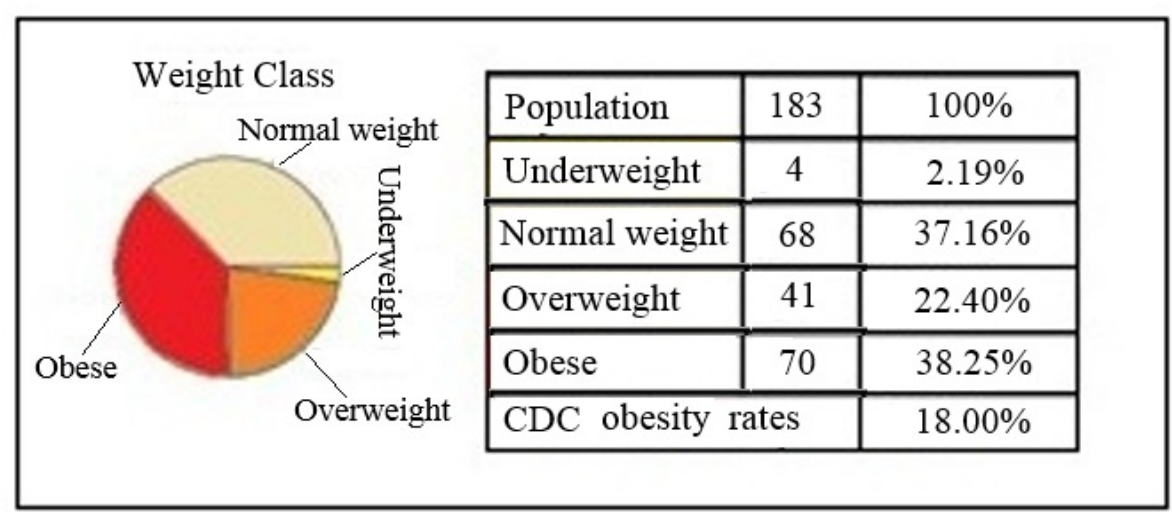

Figure 7. Total population weight-class pie chart visualizing weight dynamics

\subsection{Male Results}

Our most important results for the male categories and this paper are listed below in Table 1 . For normal weight, we saw that waist was a very poor weight indicator (p-value $<0.001$ ). Calf skin-fold appeared to be a somewhat better predictor of normal weight than triceps skin-fold, although both measures were strong ( $p$-value of 0.078 and 0.066 , respectively). For obese weight, triceps skin-fold appeared to be a better weight indicator than calf skin-fold (p-value of 0.69 and 0.57 , respectively). However, we found that waist was still not a reliable predictor for obesity and overweight (p-value $<$ 0.001). Due to the small sample of female subjects in our study population, we were unable to separate them reasonably into unique weight categories like normal and obese.

Figure 2.0-2.1 shows that the calf skin-fold and triceps-skin fold measures appear to be the most consistent for predicting weight classes for male Native American adolescents, and that the waist seems to be the least consistent weight class predictor. Figures 3.0-3.2 confirms these calculations by further providing visuals that combined weighted means with their respective density plots. In effect, we used our NHANESIII data in order to discover how atypical our anthropometric Native American adolescent factors were when compared to these national statistics (see Table 2).

Afterwards, we compared our data with NHANESIII surveys and discovered three measures of statistical interest for normal and obese weight male Native American Adolescents: waist, triceps skin-fold and calf skin-fold. Then, we gathered measures of center to indicate which anthropometric indicators were best-suited to measuring obesity in male Native American adolescents, and to identify which deviated most significantly from accepted norms (see Figure 3.0-3.2). 
Table 1. Discovering normality for anthropometric measures with Shapiro-Wilks analysis

\begin{tabular}{lcc}
\hline Anthropometrics sorted by weight class & Shapiro-Wilks & p-value \\
\hline Normal weight class & & \\
Waist mean & 0.8286 & $<.001$ \\
Triceps skin-fold & 0.9313 & $0.0664^{*}$ \\
Calf skin-fold & 0.9341 & $0.0782^{*}$ \\
Obese weight class & & \\
Waist mean & 0.8257 & $<.001$ \\
Triceps skin-fold & 0.9741 & $0.693^{*}$ \\
Calf skin-fold & 0.9696 & $0.570^{*}$ \\
\hline
\end{tabular}

Table 2. Male Anthropometrics compared to NHASES

\begin{tabular}{lcccccc}
\hline \multicolumn{1}{c}{ Anthropometrics } & N & Mean & S.D. & df & T & p-value \\
\hline Waist mean & 73 & 76.08 & 27.88 & 72 & -2.22 & $<.05$ \\
Triceps skin-fold & 70 & 19.74 & 7.18 & 69 & 7.79 & $<.001$ \\
Calf skin-fold & 70 & 30.90 & 5.59 & 69 & -9.58 & $<.001$ \\
\hline
\end{tabular}

Table 3. Male NHANESIII data, ages 14-18

\begin{tabular}{lccc}
\hline \multicolumn{1}{c}{ Anthropometrics } & $\mathbf{N}$ & Weighted Mean & Pooled S.D. \\
\hline Waist mean & 821 & 83.33 & 3.62 \\
Triceps skin-fold & 811 & 13.05 & 0.53 \\
Calf skin-fold & 1373 & 37.30 & 0.92 \\
\hline
\end{tabular}

Our analysis sought to answer this question: what is the best anthropometric indicator to measure obesity within our sample population? For male Native American adolescents, our analysis was two- fold. Our first step analyzed the normal weight class. First, we had to choose between the triceps skin-fold mean and the calf skin-fold mean (p-values of 0.066 and 0.078 , respectively). Although it appeared more likely that the calf skin-fold mean was derived from a normal population, our z-scores told us another story. Between these measures we had a $\mathrm{z}$-value of -1.03 for the calf skin-fold mean versus a $\mathrm{z}$-value of 0.60 for the triceps skin-fold mean based upon NHANESIII. Due to these results, we close to favor the calf skin-fold measure as the best assessor of normal weight for male Native American adolescents, and the triceps skin-fold measure as the best assessor of obese weight for male Native American adolescents.

\section{CONCLUSION}

In summary, this study was designed to explore which anthropometric indicators best predicted obesity in male Native American adolescents. Under the conditions of our study, we found the calf skin- fold measure to be the best indicator of normal weight for our population, and the triceps skin-fold mean to be the best indicator of obesity for our population. In addition to this pilot study, more research exploring the reliability of NHANESIII data to assess obesity in Native American adolescents is recommended in order to confirm our discovery.

\section{ACKNOWLEDGEMENTS}

Special thanks to Mr. Roland Doepner, Sherman Indian High School; Hannah Te Stipek, Washington State University; and Dr. Wayne Fletcher, California Baptist University; for their collaboration and support.

\section{REFERENCES}

[1] World Health Organization, "Diet, nutrition and the prevention of chronic diseases. Report of a WHO Study Group, Geneva," WHO Technical Report Series, no. 797 -TRS 797, 1990.

[2] Ekonoyan, G., "A history of obesity, or how what was good became ugly and then bad", Adv Chronic Kidney Dis, vol/issue: 13(4), pp. 421-427, 2006.

[3] Fryar CD., Gu Q., Ogden CL., "Anthropometric reference data for children and adults: United States, 20072010. National Center for Health Statistics", Vital Health Stat., vol/issue: 11(252), 2012.

[4] Brancati FL., Kao WH., Folsom AR., Watson RL., Szklo M., "Incident type 2 diabetes mellitus African American and white adults: the Atherosclerosis Risk in Communities Study", JAMA, vol. 283, pp. 2253-2259, 2000.

[5] Hedley AA., Ogden CL., Johnson CL., et al., "Prevalence of overweight and obesity among US children, 
adolescents, and adults, 1999-2002”, JAMA, vol. 291, pp. 2847-2850, 2004.

[6] Ogden CL., Carroll MD., Kit BK., Flegal KM., "Prevalence of childhood and adult obesity in the United States, 2011-2012", Journal of the American Medical Association, vol/issue: 311(8), pp. 806-814, 2014.

[7] McDowell M., Fryar CD., Ogden CL., Flegal KM., "Anthropometric reference data for children and adults: United States, 2003-2006. National Center for Health Statistics,” Vital Health Stat., vol/issue: 10(145), 2008.

[8] Clark VR., Greenberb B., Harris TS., Carson BL., "Body Mass Index and waist circumference predictors of cardiovascular risk in African Americans", Ethnic Dis., vol/issue: 22(2), pp. 1627, 2012.

[9] R Core Team, "R: A language and environment for statistical computing," $R$ Foundation for Statistical Computing, 2014.

[10] Hedderson M., Ehrlich S., Sridhar S., Darbinian J., Moore S., Ferrara A., "Racial/Ethnic Disparities in the Prevalence of Gestational Diabetes by BMI", Diabetes Care, vol. 35, pp. 1492-8, 2012.

[11] Zilanawala A., Davis-Kean P., Nazroo J., Sacker A., Simonton S., Kelly Y., "Race/ethnic disparities in early childhood BMI, obesity and overweight in the United Kingdom and United States", International Journal of Obesity, vol. 39, pp. 520-529, 2014.

\section{BIOGRAPHIES OF AUTHORS}

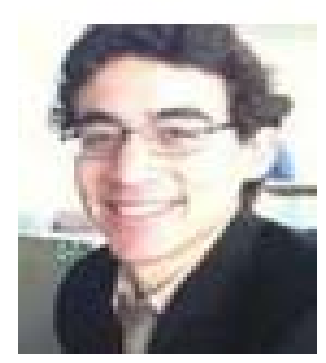

Benjamin Knisley is a senior completing his B.S. in Applied Statistical Analysis at California Baptist University. In 2014, he received the award for excellence in statistics research from the department of natural and mathematical sciences. He plans to begin his M.S. in Applied Mathematics at California Baptist University in September, 2015.

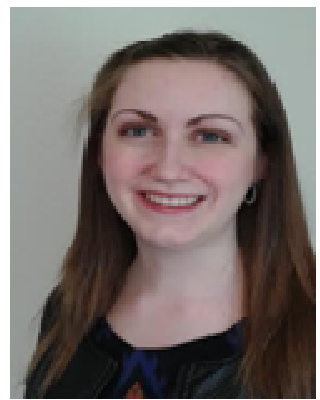

Grace Crosby is a senior completing her B.S. in Healthcare Administration at California Baptist University. In 2014, she received the Scholarship of Excellence award from the Association of Schools of Allied Health Professions (ASAHP). She plans to begin her MPH with an emphasis in Food, Nutrition and Health at California Baptist University in September, 2015.

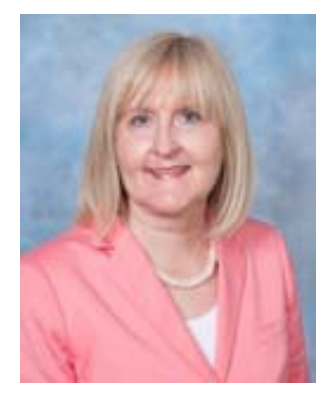

Margaret Barth, PhD, MPH, MBA, is Program Director of Nutrition and Food Sciences and Professor of Nutrition and Food Sciences at California Baptist University. Previously, she was Associate Professor of Nutrition and Food Science at the University of Kentucky, Lexington. Dr. Barth received her PhD in Food Science and Human Nutrition from the University of Illinois.

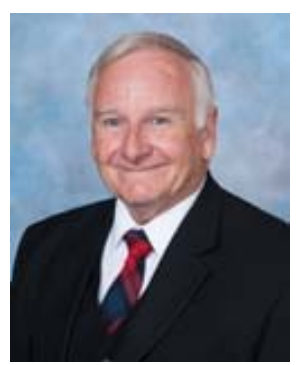

Linn Carothers, $\mathrm{PhD}$, is Program Director of Applied Statistics and Physics and Professor of Mathematics at California Baptist University. Dr. Carothers received his PhD in Educational Psychology and Statistics from the University of Southern California. 